\title{
O PROBLEMA DE DIMENSIONAMENTO DE LOTES COM PLANTAS FLEXÍVEIS E CUSTO DE TRANSPORTE ${ }^{1}$
}

\author{
Samanta Bellei Teixeira ${ }^{a}$, Silvio Alexandre de Araujo ${ }^{a}$, Diego Jacinto Fiorotto ${ }^{\text {b* }}$ \\ ${ }^{a}$ Departamento de Matemática \\ Universidade Estadual Paulista - UNESP, São José do Rio Preto-SP, Brasil \\ ${ }^{\mathrm{b}}$ Faculdade de Ciências Aplicadas \\ Universidade Estadual de Campinas - UNICAMP, Limeira-SP, Brasil
}

Recebido 05/02/2020, aceito 03/05/2020

\begin{abstract}
RESUMO
O problema de dimensionamento de lotes com plantas flexíveis e custo de transporte consiste em definir quais plantas deverão ser flexíveis para produzir determinados produtos, em determinar as quantidades que devem ser produzidas, bem como, para quais clientes estes produtos deverão ser entregues de forma a suprir as demandas predeterminadas, em um horizonte de planejamento finito. O objetivo consiste em minimizar os custos de produção, preparo, estoque, hora-extra, flexibilidade e transporte. Neste trabalho propõem-se um modelo matemático para este problema, além de uma análise computacional a fim de verificar se, com um orçamento de flexibilidade predefinido, pode-se obter benefícios semelhantes aos da flexibilidade total (todas as plantas podem produzir todos os produtos). Os testes computacionais indicam que é possível obter bons resultados com flexibilidade reduzida.
\end{abstract}

Palavras-chave: Problema de dimensionamento de lotes, Problema do transporte, Problemas integrados, Plantas flexíveis.

\begin{abstract}
The lot sizing problem with flexible plants and transport costs consists in defining which plants should be flexible to produce certain products, to determine the quantities that should be produced in each plant and to which customers these products should be delivered in order to meet predetermined demands, in a finite planning horizon. The aim is to minimize production, setup, inventory, overtime, flexibility and transportation costs. In this paper, we propose a mathematical formulation for this problem, as well as a computational analysis, in order to verify if, with a predetermined budget, it is possible to obtain similar benefits to the total flexibility (all plants can produce all products). The computational experiments indicate that it is possible to obtain good results with reduced flexibility.
\end{abstract}

Keywords: Lot sizing problem, Transportation problem, Integrated problems, Flexible plants.

*Autor para correspondência. E-mail: fiorotto@unicamp.br

DOI: 10.4322/PODes.2020.003

${ }^{1}$ Todos os autores assumem a responsabilidade pelo conteúdo do artigo. 


\section{Introdução}

O gerenciamento em uma indústria, que tem por objetivo a conversão de matéria prima em produto final, deve seguir uma linha de qualidade eficaz, tendo a preocupação de atender uma demanda estabelecida com o menor custo possível. A decisão da melhor forma de se realizar a disposição dos recursos provenientes de uma indústria pode ser dividida em três tipos de planejamentos diferentes, que são: estratégico, tático e operacional.

De forma geral, o planejamento estratégico visa resultados a longo prazo, auxiliando na definição dos objetivos e estratégias para alcançá-los. Por sua vez, o planejamento tático tem como enfoque o planejamento a médio prazo e o auxílio das atividades decorrentes dos vários departamentos da indústria. Por fim, o planejamento operacional está voltado para a realização das atividades determinadas, e está relacionado com o que fazer e o como fazer para se alcançar os objetivos desejados.

O problema de dimensionamento de lotes situa-se nos níveis tático/operacional e consiste em determinar a quantidade de itens a serem produzidos ao longo de um horizonte de tempo finito, em que é necessário que uma certa demanda predeterminada seja atendida utilizando-se uma única ou várias máquinas. Os custos envolvidos neste problema são de produção, estoque e preparação de máquinas. Este problema tem origem econômica, e o objetivo consiste em minimizar os custos relacionados à produção.

Com a evolução dos processos produtivos, bem como, das pesquisas relacionadas, nos último anos, as decisões referentes ao problema de dimensionamento de lotes vem sendo tomadas de maneira integrada à outras decisões que também são importantes para o meio industrial. Além disso, recentemente, tem surgido estudos que consideram diferentes fontes de flexibilidade aplicadas ao problema de dimensionamento de lotes (por exemplo, Fiorotto et al. (2018) para flexibilidade de máquinas e Belo-Filho et al. (2014), Fiorotto et al. (2017) e Fiorotto et al. (2019) para flexibilidade na preparação de máquinas) fazendo com que o problema se torne mais parecido com o que ocorre na prática.

Neste artigo o conceito de flexibilidade está relacionado com a decisão de quais produtos podem ser produzios em cada uma das plantas produtivas (Jordan e Graves, 1995). Uma vez que a flexibilidade total de plantas (todas as plantas podem produzir todos os produtos) pode ser muito cara, ou até mesmo impraticável, é importante estudar maneiras de implementar uma quantidade limitada de flexibilidade (quais produtos devem ser produzidos em cada uma das plantas) para balancear os custos e benefícios. Seguindo esta tendência, o presente trabalho trata do problema de dimensionamento de lotes, o qual é integrado ao problema do transporte considerando um ambiente de produção constituído de plantas flexíveis com capacidade limitada. Diversos produtos são demandados por diferentes clientes e devem ser produzidos pelas plantas. O transporte dos produtos produzidos até os clientes é realizado levando em consideração um custo referente aos valores gastos com combustível, cobranças de pedágios, etc.

Embora a flexibilidade seja, no geral, uma decisão estratégica tipicamente analisada em um contexto com demanda estocástica, o estudo apresentado por Fiorotto et al. (2018) mostra que a flexibilidade pode ter um valor significativo em um ambiente de planejamento determinístico de curto prazo. Neste contexto, a decisão de configuração de flexibilidade torna-se uma decisão tático/operacional que é tomada no início do horizonte de planejamento quando a demanda é conhecida. Assim, a configuração de flexibilidade permanece fixa para o horizonte de planejamento atual. Esta é, portanto, uma situação diferente quando comparada com o cenário estocástico onde as decisões de flexibilidade devem ser tomadas sem conhecer a demanda exata. Tal situação ocorre, por exemplo, na indústria de semicondutores, onde as máquinas devem ser qualificadas antes de serem capazes de produzir certos produtos, e essas decisões de qualificação são reavaliadas periodicamente (Johnzén et al., 2011; Rowshannahad et al., 2015). A qualificação de uma máquina para um produto específico pode ser uma tarefa muito cara e, portanto, é feita periodicamente (Rowshannahad et al., 2015). 
Com o intuito de estudar o valor da flexibilidade de máquinas, Fiorotto et al. (2018) mostra que com uma quantidade limitada de flexibilidade é possível obter praticamente os mesmos benefícios da flexibilidade total para o problema de dimensionamento de lotes com máquinas paralelas. Neste trabalho estende-se o estudo apresentado por Fiorotto et al. (2018) para o problema integrado de dimensionamento de lotes e transporte. A decisão de quais plantas flexibilizar para serem capazes de produzir certos tipos de produtos agora também leva em consideração os custos de transporte e, portanto, a dispersão geográfica das plantas e dos clientes. O desafio é analisar a quantidade ideal de flexibilidade necessária, com o intuito de minimizar custos e atender toda a demanda dos clientes. Desta forma, do ponto de vista da prática industrial as análises apresentadas neste artigo se justificam devido à revelância do estudo de problemas integrados e a importância da consideração de flexibilidade, que advém do crescente estímulo que as indústrias têm recebido para otimizar seus processos produtivos e, uma visão flexível do processo produtivo pode auxiliar de forma significativa o processo de decisão.

As principais contribuições deste artigo são: propor uma formulação matemática para o problema de dimensionamento de lotes com custos de transporte que permite determinar a melhor configuração de flexibilidade para um orçamento predeterminado; realizar, através de um estudo computacional, uma análise sobre o valor da flexibilidade para o problema abordado, com o intuito de verificar se com uma quantidade pequena de flexibilidade é possível encontrar benefícios similares aos da flexibilidade total.

As outras seções deste trabalho estão divididas da seguinte maneira: na Seção 2, são apresentados estudos sobre flexibilidade e sobre os problemas de dimensionamento de lotes com várias máquinas e com várias plantas que estão relacionados com este trabalho; na Seção 3, uma formulação matemática para o problema de dimensionamento de lotes com custos de transporte e plantas flexíveis é proposta; na Seção 4, apresenta-se a geração de dados e os resultados computacionais e, na Seção 5, são realizadas as conclusões e as ideias para trabalhos futuros.

\section{Trabalhos Correlatos}

Embora o problema de dimensionamento de lotes com plantas flexíveis e custo de transporte foi apenas recentemente abordado na literatura (Teixeira, 2019), o problema de dimensionamento de lotes com várias máquinas e com várias plantas, bem como, alguns conceitos de flexibilidade foram abordados em diversos estudos em diferentes problemas de planejamento da produção. Na Seção 2.1, apresenta-se uma revisão sobre alguns estudos que consideram o problema de dimensionamento com várias máquinas e com várias plantas e estão relacionados com o problema estudado. Em seguida, apresenta-se alguns estudos relacionados a flexibilidade.

\subsection{Problemas de Dimensionamento de Lotes com Várias Máquinas e com Várias Plantas}

O problema de dimensionamento de lotes com várias máquinas aparece em diversas aplicações industriais (Jans e Degraeve, 2004). Existem vários trabalhos que consideram o problema de dimensionamento de lotes com várias máquinas e flexibilidade total. Para o problema com várias máquinas idênticas, Carreno (1990) propõe uma heurística para o problema de dimensionamento de lotes considerando tempos de preparação de máquinas e demanda constante. Jans (2009) propõe uma formulação baseada no problema do caminho mínimo e apresenta novas restrições para quebrar a simetria presente no problema. Os resultados computacionais mostram que os tempos computacionais diminuem significativamente ao considerar tais restrições.

Considerando o problema de dimensionamento de lotes com máquinas não relacionadas, Toledo e Armentano (2006) relaxa as restrições de capacidade e desenvolvem uma heurística Lagrangiana para resolver o problema. Uma solução inicial é obtida através da minimização do problema Lagrangiano seguido por um passo de melhoria na tentativa de melhorar/tornar factível a solução inicial. Mateus et al. (2010) propõem um processo iterativo para gerar planos de produção que levam em consideração restrições de sequênciamento. Os resultados computacionais mos- 
tram que a abordagem utilizada é eficiente para resolver problemas com até 50 itens. Fiorotto e de Araujo (2014) propõem uma heurística Lagrangiana utilizando a reformulação do problema como um problema do caminho mínimo e, diferente da maioria dos trabalhos que aplicam a decomposição por itens, os autores utilizam a decomposição por períodos. Os resultados mostram que o método é eficiente tanto para a qualidade dos limitantes inferiores quanto para gerar soluções factíveis. Com o objetivo de obter limites inferiores de alta qualidade para o problema, Fiorotto et al. (2015) utilizam métodos híbridos que combinam a decomposição de Dantzig-Wolfe e relaxação Lagrangiana. A estratégia abordada foi comparada com duas heurísticas Lagrangianas propostas na literatura e os experimentos computacionais mostram que os métodos híbridos produzem limites inferiores e soluções factíveis melhores do que os encontrados com as heurísticas Lagrangianas. Wu et al. (2018) propõem diferentes formulações matemáticas para o problema e analisa as decomposições por itens e por períodos destas formulações. Por fim, um estudo sobre a eficiência dos limitantes inferiores obtidos através destas decomposições é realizado. Vincent et al. (2020) propõem uma metaheurística para resolver este problema. A abordagem utilizada baseia-se na relaxação das restrições de capacidade a fim de explorar o conjunto de soluções para encontrar mais soluções factíveis. Os resultados mostram que a abordagem utilizada é eficiente em termos de qualidade de solução.

O problema de dimensionamento de lotes com várias plantas consiste em uma extensão natural do problema com várias máquinas e também aparece em várias aplicações práticas. Em geral, considera-se que cada uma das plantas tem uma demanda própria e existe a possibilidade de transferência de produção entre as plantas (Sambasivan e Yahya, 2005). Um dos primeiros estudos que consideram um ambiente de produção composto de várias plantas é apresentado em Bhatnagar et al. (1993), em que o objetivo é coordenar os planos de produção e estoque em todas as plantas de modo que o desempenho global e a competitividade da empresa seja melhorada. Em de Matta e Miller (2004) a decisão de dimensionamento de lotes é integrada às decisões de transporte de itens entre as plantas de uma mesma indústria de forma que algumas plantas produzem produtos intermediários e outras os produtos finais. O objetivo principal dos autores consiste em compreender as relações existentes nas decisões de mudança de capacidade, alteração dos custos, escolhas de transporte, dentre outros. Guimaraes et al. (2012) apresentam uma formulação para um problema com várias plantas em uma indústria de bebidas. Os autores estudam o planejamento das operações que definem o escalonamento e o dimensionamento da produção, em que o objetivo é satisfazer a procura estimada minimizando os custos de produção, horas extras e transferência. Os resultados obtidos mostram a interdependência entre o planejamento da produção e a distribuição. Melega et al. (2013) analisam a eficiência de diferentes reformulações que baseiamse no problema do caminho mínimo e localização de facilidades. A partir da análise dos resultados computacionais, conclui-se que todas as reformulações propostas obtiveram resultados melhores do que a formulação original para a maioria dos exemplares analisados.

Mais recentemente, Carvalho e Nascimento (2016) abordam este problema considerando que todas as plantas produzem os mesmos itens (cada uma das plantas com uma única máquina) e que as demandas devem ser atendidas sem atraso. Heurísticas Lagrangianas são propostas e os experimentos computacionais mostram que a qualidade das soluções encontradas com os métodos propostos superam significativamente a melhor heurística da literatura. Considerando o problema com várias plantas e preparações de máquinas carry-over, Carvalho e Nascimento (2018) aplicam uma metaheurística denominada método kernel search para resolver este problema. Os autores observam que o espaço de soluções factíveis se torna significantemente maior considerando a possibilidade de preparações de máquinas carry-over. Além disso, a redução do número de preparações de máquinas é siginificante, principalmente para instâncias com custos de preparação alto. 


\subsection{Flexibilidade em Ambientes Industriais}

Embora alguns estudos sobre os principais conceitos e o potencial de considerar flexibilidade em ambientes industriais, principalmente para eficácia de processos de fabricação com uma quantidade considerável de incertezas, já tivessem sido propostos por alguns autores (destacase, por exemplo, Gerwin (1993)), Jordan e Graves (1995) foi o primeiro artigo a considerar a eficácia da utilização de uma quantidade limitada de flexibilidade de máquinas, mostrando que uma configuração encadeada de produtos e plantas (princípio da cadeia) pode ter um desempenho muito eficaz quando comparado ao caso com flexibilidade total em termos de rendimento e uso da capacidade. O princípio da cadeia pode ser definido como um grupo de itens e máquinas (plantas) que estão todos ligados, direta ou indiretamente, por decisões de atribuções de produtos. Em termos de teoria dos grafos uma cadeia é um grafo conexo minimal (ver parte (b) da Figura 1). Dentro de uma cadeia, um caminho pode ser traçado a partir de qualquer item ou planta para qualquer outro item ou planta através das ligações de atribuição do produto. O segredo por trás de encadeamento é que todos os itens da cadeia compartilham de forma eficaz toda a capacidade das plantas presentes na cadeia. O conceito de encadeamento é uma das configurações de flexibilidade parcial mais utilizadas para estudos na literatura, em particular, para estudos em industrias automotivas (Graves e Tomlin, 2003).

Após os estudos apresentados por Jordan e Graves (1995), diversos trabalhos foram propostos para avaliar o valor da flexibilidade de máquinas em um contexto constituído por demandas estocásticas. Koste e Malhotra (1999) desenvolvem fundamentos teóricos para construir medidas gerais para quantificar o conceito de flexibilidade. Para tanto, o estudo define dez dimensões de flexibilidade e suas características. Uma hierarquia que propõe o relacionamento entre essas dimensões também é apresentada. Graves e Tomlin (2003) estendem o trabalho de Jordan e Graves (1995) e desenvolvem novas estratégias para a implementação da flexibilidade. Bertrand (2003) apresenta uma revisão bibliográfica sobre os conceitos de flexibilidade e discute três características da flexibilidade para a configuração da cadeia de suprimentos: flexibilidade de volume, flexibilidade de mistura de produtos e flexibilidade de novos produtos. O estudo demonstra as relações entre investimentos em recursos dedicados e flexíveis, e sua dependência do nv́el de incerteza da demanda. Por fim, os autores discutem algumas perspectivas futuras para análise do valor da flexibilidade. Muriel et al. (2006) mostram que praticamente todos os benefícios com aumento de vendas podem ser encontrados com o princípio da cadeia. Além disso, a quantidade de estoque é reduzida significativamente a medida que mais flexibilidade é adicionada ao sistema. Portanto, de acordo com os autores, se os custos de estoques são caros, adicionar flexibilidade de forma eficiente, torna o processo produtivo mais econômico.

Embora vários estudos mostrem que o princípio da cadeia apresenta boa performance, especialmente para sistemas homogêneos, existem também estudos que demonstram que nem sempre é esse o caso. Mak e Shen (2009) mostram que o princípio da cadeia as vezes tem um desempenho insatisfatório quando a demanda e os custos de produção não são homogêneos. Gurumurthi e Benjaafar (2004) apresentam resultados numéricos para sistemas de filas e concluem que, em sistemas assimétricos, existe sempre uma configuração não encadeada que funciona melhor do que o princípio da cadeia.

No contexto do problema de dimensionamento de lotes, alguns trabalhos consideram sistemas produtivos em que não se tem flexibilidade total. Jans e Degraeve (2004) discutem um problema com flexibilidade parcial aplicado à indústria de pneus, onde nem todos os pneus podem ser produzidos em todos os tipos de aquecedores. Xiao et al. (2015) propõem uma heurística Lagrangiana para o problema com máquinas paralelas capacitadas com escalonamento, onde nem todas as máquinas são capazes de produzir todos os itens.

Em Fiorotto et al. (2018) é analisado o valor da flexibilidade do processo no contexto de um problema de dimensionamento de lote determinístico de curto prazo, onde a decisão de flexibilidade é tomada no início do horizonte de planejamento. Para realizar as análises, os autores propõem dois modelos, um modelo para determinar a melhor cadeia longa, e outro modelo 
de otimização que considera a possibilidade de investir em flexibilidade e determina a melhor configuração de flexibilidade com um determinado orçamento disponível. Os resultados computacionais obtidos mostram que quase todos os benefícios da flexibilidade do processo são obtidos ao adicionar uma pequena quantidade de flexibilidade (ligações entre máquina e itens). Além disso, os autores observam que o princípio da cadeia obtém praticamente todos os benefícios da flexibilidade total para instâncias em que a capacidade não é muito apertada e para sistemas homogêneos. No entanto, para problemas em que a capacidade é muito apertada ou o sistema não é homogêneo este princípio não apresenta bons resultados.

Devido ao crescente interesse em adicionar aos modelos aspectos que ocorrem na prática, algumas pesquisas foram desenvolvidas com o objetivo de adicionar diferentes tipos de flexibilidades a alguns problemas. De fato, a literatura sobre flexibilidade de máquinas aborda um amplo espectro de questões que vão desde as decisões estratégicas de planejamento de capacidade (Fine e Freund, 1990; Jordan e Graves, 1995) até detalhadas questões de operações de número de troca de ferramentas (Tang e Denardo, 1988). Pérez-Pérez et al. (2018) propõem uma revisão sistemática recente da literatura sobre flexibilidade. O estudo utiliza 284 artigos publicados até 2017 e apresenta as principais questões que foram investigadas na literatura. Por fim, os autores apresentam várias oportunidades de pesquisas futuras. Observa-se que dentre as oportunidades apresentadas, este artigo se enquadra no estudo de desenvolvimento e análise dos benefícios de novas configurações de flexibilidade. Por fim, destaca-se ainda existem poucos estudos com o intuito de determinar os benefícios da flexibilidade no contexto de problemas de dimensionamento de lotes.

\section{Definição do Problema e Formulação Matemática}

Neste seção, propõem-se uma formulação matemática para o problema integrado de dimensionamento de lotes e transporte considerando a possibilidade de uma quantidade limitada de flexibilidade. Esta formulação estende as ideias proposta por Fiorotto et al. (2018) para o problema abordado e considera a possibilidade de investir em flexibilidade. Desta forma, o modelo permite determinar a melhor configuração de flexibilidade para um orçamento global de investimento predeterminado. Observa-se ainda que o ambiente de produção é constituído por várias plantas interligadas, que devem atender a demanda de vários clientes que ocorrem em um horizonte de planejamento finito dividido por períodos.

Produzir vários produtos em um horizonte de planejamento finito é comum em diversos contextos industriais, onde minimizar gastos referentes a produção, estoque e preparação é o principal objetivo. Observa-se que o transporte de determinada produção pode afetar diretamente na rentabilidade de uma indústria, desta forma algumas preferem realizar, além do planejamento de produção, o planejamento do transporte dos produtos até o seu cliente, o que pode gerar uma economia significativa no custo final dos produtos. Observa-se ainda que esta economia pode ser alcançada a partir de modelos que auxiliam nas tomadas de decisão de fluxo do produto a ser entregue de fornecedor para cliente.

Investir em flexibilidade é disponibilizar a possibilidade de uma planta produzir um determinado produto ou não, tal decisão é tomada no início do horizonte de planejamento e determina a forma com que a produção e o transporte serão direcionados durante todos os períodos que compõem o horizonte de planejamento. A Figura (1) ilustra o problema de dimensionamento de lotes com plantas flexíveis e custo de transporte considerando três configurações de flexibilidade para um problema com 4 itens que devem ser produzidos em 4 plantas e entregues a $p$ clientes. A primeira configuração (caso (a)) é o caso dedicado, em que cada uma das plantas produz exatamente um único item. No caso (b) e (c), ligações adicionais entre itens e plantas são inseridas aumentando assim a flexibilidade do sistema. No caso (b) apresenta-se a regra da cadeia em que a quantidade de ligações adicionais (em relação ao caso dedicado) corresponde ao número de itens do problema. No caso (c) têm-se a flexibilidade total em que todas as ligações possíveis entre 
plantas e máquinas estão presentes no sistema. Observa-se que o problema considera que todas as plantas podem entregar os produtos para todos os $p$ clientes (com os devidos custos de transporte), de forma que a decisão de quais itens devem ser produzidos em cada uma das plantas leva em consideração os custos de transporte.

Figura 1: Configurações de flexibilidade para um problema com 4 itens, 4 plantas e $p$ clientes.

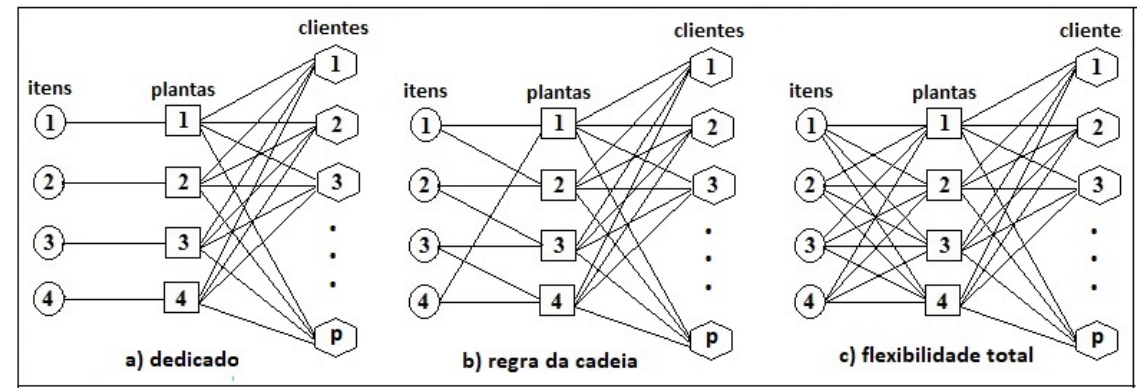

Fonte: Elaborado pelos autores.

As demais hipóteses consideradas no problema são:

1) o número de itens é sempre igual ao número de plantas (problema balanceado);

2) a quantidade de flexibilidade do problema é determinada em relação a quantidade de ligações entre itens e plantas disponíveis;

3) os custos e tempos de produção e preparação não dependem da sequência;

4) considera-se a possibilidade de hora extra em cada uma das plantas;

5) as demandas dos clientes são independentes;

6) considera-se que o produto fica estocado na planta e é entregue ao cliente no período demandando.

Dessa forma, o modelo matemático proposto une dois aspectos relevantes e impactantes quando pensamos nos interesses de uma indústria, a produção e o transporte de seus produtos confeccionados. A seguir apresenta-se a formulação proposta para o problema de dimensionamento de lotes com plantas flexíveis e custo de transporte. Observa-se que tal formulação é uma extensão da formulação proposta em Fiorotto et al. (2018). No entanto, diferente de Fiorotto et al. (2018), que utiliza uma formulação clássica, neste trabalho considera-se uma reformulação baseada no problema do transporte (Denizel e Süral, 2006).

Para a formulação matemática do problema considere os seguintes parâmetros:

$I=\{1, \ldots, n\}: \quad$ conjunto de produtos;

$J=\{1, \ldots, r\}: \quad$ conjunto de plantas;

$K=\{1, \ldots, p\}: \quad$ conjunto de clientes;

$T=\{1, \ldots, m\}:$ conjunto de períodos;

$d_{i t}^{k}$ :

demanda do produto $i$ para o cliente $k$ no período $t$;

$h c_{i t}$ :

custo unitário de estoque do produto $i$ no período $t$;

$e c_{j t}$ :

custo unitário da utilização de hora extra na planta $j$ no período $t$;

$s c_{i j t}$ :

custo de preparo da planta $j$ para o produto $i$ no período $t$;

$v c_{i j t}$ :

custo de produção da planta $j$ para o produto $i$ no período $t$;

$s t_{i j t}$ :

tempo de preparo da planta $j$ para o produto $i$ no período $t$;

$v t_{i j t}$ : $\quad$ tempo de produção da planta $j$ para o produto $i$ no período $t$;

$s d_{i t}$ : $\quad$ soma das demandas do produto $i$ do período $t$ até o final do horizonte de planejamento;

$\operatorname{cap}_{j t}: \quad \quad$ capacidade (em unidade de tempo) da planta $j$ no período $t$;

$f c_{i j}$ : $\quad$ custo para tornar a planta $j$ flexível para produzir o item $i$; 
Fmax: $\quad$ orçamento disponível para investir em flexibilidade;

$t c_{i j}^{k}$ : $\quad$ custo unitário de transporte para enviar uma unidade do produto $i$ da planta $j$ para o cliente $k$;

$\operatorname{Cost}_{i j t l}^{k}$ : $\quad$ custo unitário da planta $j$ para a produção, estoque e transporte do produto $i$ no período $t$ para satisfazer a demanda do cliente $k$ no período $l(t \leq l)$ e $\operatorname{Cost}_{i j t l}^{k}=v c_{i j t}+t c_{i j}^{k}+\sum_{s=t+1}^{l} h c_{i s}$.

As variáveis de decisão são definidas por:

$y_{i j t}$ : variável binária, indicando a produção ou não do produto $i$ na planta $j$ no período $t$;

$z_{i j}$ : variável binária, indicando se a planta $j$ é flexível para produzir ou não o produto $i$;

$x_{i j t l}^{k}$ : número de unidades produzidas do produto $i$ na planta $j$ no período $t$ para satisfazer a demanda do cliente $k$ no período $l$, com $t \leq l$;

$o v_{j t}$ : número de unidades de hora extra utilizados na planta $j$ no período $t$.

Desta forma, a formulação do problema é dada por:

$$
\min \sum_{j=1}^{r} \sum_{i=1}^{n} \sum_{t=1}^{m} s c_{i j t} y_{i j t}+\sum_{k=1}^{p} \sum_{i=1}^{n} \sum_{j=1}^{r} \sum_{t=1}^{m} \sum_{l=1}^{m} \operatorname{Cost}_{i j t l}^{k} x_{i j t l}^{k}+\sum_{j=1}^{r} \sum_{t=1}^{m} e c_{j t} \text { ov } v_{j t}
$$

Sujeito a :

$$
\begin{array}{lr}
\sum_{j=1}^{r} \sum_{t=1}^{l} x_{i j t l}^{k}=d_{i l}^{k} & \forall k \in K, \forall i \in I, \forall l \in T \\
x_{i j t l}^{k} \leq s d_{i t} y_{i j t} & \forall k \in K, \forall i \in I, \forall j \in J, \forall t \in T, \forall l \in T \\
\sum_{i=1}^{n}\left(s t_{i j t} y_{i j t}+\sum_{k=1}^{p} \sum_{l=t}^{m} v t_{i j t} x_{i j t l}^{k}\right) \leq c a p_{j t}+o v_{j t} & \forall j \in J, \forall t \in T \\
y_{i j t} \leq z_{i j} & \forall i \in I, \forall j \in J, \forall t \in T \\
\sum_{i=1}^{n} \sum_{j=1}^{r} f c_{i j} z_{i j} \leq F \max & \\
y_{i j t} \in\{0,1\}, \quad z_{i j} \in\{0,1\} & \forall i \in I, \forall j \in J, \forall t \in T \\
x_{i j t l}^{k} \geq 0, o v_{j t} \geq 0 & \forall k \in K, \forall i \in I, \forall j \in J, \forall t \in T, \forall l \in T
\end{array}
$$

A função objetivo (1) minimiza custos de preparação, transporte, produção, estoque e hora extra. As restrições (2) asseguram que toda a demanda seja satisfeita no horizonte de planejamento. Em seguida, as restrições (3) garantem a preparação da planta antes da efetiva produção, já as restrições (4) são as restrições de capacidade do problema e considera a possibilidade de utilização de hora extra a um certo custo na função objetivo. As restrições (5) garantem que a planta possa produzir um produto apenas se ela for flexível para tal produção. Nas restrições (6) é garantido que o orçamento disponível para investir em flexibilidade não seja extrapolado. Finalmente, as restrições (7) e (8) definem o domínio das variáveis.

\section{Análise de Flexibilidade}

Nesta seção, inicialmente, descreveremos a maneira como os dados serão gerados, para, posteriormente, apresentarmos os resultados computacionais que nos permitirão analisar o impacto da flexibilidade das plantas. 


\subsection{Geração dos Dados}

A geração dos dados utilizados nos testes computacionais deste trabalho consiste em uma adaptação de um conjunto de dados utilizado em Fiorotto et al. (2018), o qual foi adaptado de um conjunto de dados padrão proposto por Trigeiro et al. (1989).

Os dados utilizados consistem em 20 instâncias com 4 produtos e 15 períodos (F1-F20 (4 produtos)) e 20 instâncias com 6 produtos e 15 períodos (F1-F20 (6 produtos)). Para cada uma das 40 instâncias foram criados problemas com plantas idênticas, isto é, as capacidades são as mesmas para cada planta e para um determinado produto, os tempos de preparação, tempos de produção e custos de preparação são os mesmos em cada uma das plantas, desde que a planta seja flexível para produzir tal produto.

A partir das instâncias propostas por Fiorotto et al. (2018), foi preciso realizar a geração de alguns dados para complementar elementos que faltam para os dados exigidos no modelo proposto neste trabalho. Inicialmente, foi acrescentado um conjunto de clientes com 12 elementos que são fixos para todas as instâncias. Além disso, o número de plantas é considerado igual ao número de produtos para cada conjunto de dados.

Os parâmetros são gerados da seguinte forma: tempos de produção $v t_{i j t}$ fixos a uma unidade de tempo, custos unitário de estoque $h c_{i t}$ com variação de 1 a 5 unidades, custos de preparação $s c_{i j t}$ variando de 400 a 1000 unidades, tempos de preparação $s t_{i j t}$ variando entre 10 e 50 unidades e custos de produção $v c_{i j t}$ nulos. São utilizados cinco níveis de capacidade, 80, 90, 100, $110 \mathrm{e}$ 120 para cada planta envolvida no processo de produção. A escolha da capacidade das plantas foi baseada em testes preliminares com o intuito de apresentar uma ampla variação de problemas para os quais as soluções tenham diferentes níveis de horas extras.

Além disso, os custos de flexibilidade $f c_{i j}$ são fixos e iguais a uma unidade monetária para todos os produtos em todas as plantas. Os custos de horas extras $e c_{j t}$ foram fixados em 300 , sendo este valor 100 vezes a média do custo de estoque. A demanda $d_{i t}^{k}$ segue os mesmos processos de geração utilizados por Trigeiro et al. (1989), em que para cada produto, a demanda média é de 100 por período, gerada a partir de uma distribuição uniforme.

Por fim, os custos de transporte $t c_{i j}^{k}$ foram calculados a partir das localizações das plantas e clientes. Um par ordenado limitado entre 0 e $1 \mathrm{em}$ um quadrado no plano cartesiano com área de $1 \mathrm{~cm}^{2}$ é gerado de forma uniforme para cada planta e cliente. Assim, os custos são calculados a partir da distância euclidiana entre cada planta e cada cliente (Melo e Wolsey, 2012).

O enfoque das análises está voltado para os valores escolhidos para o parâmetro Fmax, que consiste no orçamento total disponível para investir em flexibilidade. Um dos casos analisados será considerado com plantas dedicadas, ou seja, cada planta deverá produzir apenas um produto, assim este Fmax deve ser igual ao número de plantas e produtos, e para comparativos serão utilizados valores como $100 \%, 80 \%, 50 \%$ e $20 \%$, onde $100 \%$ significa que todas as plantas produzem todos os produtos, ou seja, flexibilidade total, enquanto os demais valores são percentuais calculados sobre esse maior orçamento para flexibilidade, reduzindo assim o nível de flexibilidade, por exemplo, no caso de $80 \%$ tomando como base a flexibilidade total, considera-se que apenas $80 \%$ das ligações podem ser utilizadas. Observa-se que para os vários níveis de flexibilidade a configuração dedicada já está preestabelecida.

Foram gerados um total de 1000 instâncias, no qual suas características são apresentadas na Tabela 1.

\subsection{Resultados Computacionais}

Os dados foram processados utilizando o solver CPLEX 12.9 com interface do software Visual Studio 2012 em linguagem algébrica C. O computador utilizado para a realização das análises possui sistema operacional Windows 10 Pro de 64 bits, processador Intel(R) Core(TM) i7-4770 CPU @ 3.40GHz e memória RAM de 16,0 GB. Os critérios de parada foram o gap igual ou inferior a $0,1 \%$ ou tempo limite de uma hora (3600 segundos). 
Tabela 1: Conjuntos de dados.

\begin{tabular}{|c|c|c|c|}
\hline Flexibilidade & Capacidade & Conjuntos & Quantidades \\
\hline \multirow{5}{*}{ Fmax 1 = Dedicado } & 80 & F1 - F20 (4 produtos) e F1 - F20 (6 produtos) & 40 instâncias \\
& 90 & F1 - F20 (4 produtos) e F1 - F20 (6 produtos) & 40 instâncias \\
& 100 & F1 - F20 (4 produtos) e F1 - F20 (6 produtos) & 40 instâncias \\
& 110 & F1 - F20 (4 produtos) e F1 - F20 (6 produtos) & 40 instâncias \\
& 80 & F1 - F20 (4 produtos) e F1 - F20 (6 produtos) & 40 instâncias \\
\hline \multirow{5}{*}{ Fmax 2=20\% } & 90 & F1 - F20 (4 produtos) e F1 - F20 (6 produtos) & 40 instâncias \\
& 100 & F1 - F20 (4 produtos) e F1 - F20 (6 produtos) & 40 instâncias \\
& 110 & F1 - F20 (4 produtos) e F1 - F20 (6 produtos) & 40 instâncias \\
& 120 & F1 - F20 (4 produtos) e F1 - F20 (6 produtos) & 40 instâncias \\
\hline Fmax 3=50\% & 80 & F1 - F20 (4 produtos) e F1 - F20 (6 produtos) & 40 instâncias \\
& 90 & F1 - F20 (4 produtos) e F1 - F20 (6 produtos) & 40 instâncias \\
& 100 & F1 - F20 (4 produtos) e F1 - F20 (6 produtos) & 40 instâncias \\
& 110 & F1 - F20 (4 produtos) e F1 - F20 (6 produtos) & 40 instâncias \\
& 120 & F1 - F20 (4 produtos) e F1 - F20 (6 produtos) & 40 instâncias \\
\hline Total & 80 & F1 - F20 (4 produtos) e F1 - F20 (6 produtos) & 40 instâncias \\
& 90 & F1 - F20 (4 produtos) e F1 - F20 (6 produtos) & 40 instâncias \\
& 100 & F1 - F20 (4 produtos) e F1 - F20 (6 produtos) & 40 instâncias \\
& 110 & F1 - F20 (4 produtos) e F1 - F20 (6 produtos) & 40 instâncias \\
& 120 & F1 - F20 (4 produtos) e F1 - F20 (6 produtos) & 40 instâncias \\
\hline Fmax 4=80\% & 80 & F1 - F20 (4 produtos) e F1 - F20 (6 produtos) & 40 instâncias \\
& 90 & F1 - F20 (4 produtos) e F1 - F20 (6 produtos) & 40 instâncias \\
& 100 & F1 - F20 (4 produtos) e F1 - F20 (6 produtos) & 40 instâncias \\
& 110 & F1 - F20 (4 produtos) e F1 - F20 (6 produtos) & 40 instâncias \\
& & F1 - F20 (4 produtos) e F1 - F20 (6 produtos) & 40 instâncias \\
\hline & & & 1000 instâncias \\
\hline
\end{tabular}

Fonte: Elaborado pelos autores.

A Tabela 2 apresenta diferentes níveis de capacidade (Cap), os limitantes superiores (LS) e a utilização de capacidade (UC) encontrados para todos os níveis de flexibilidade. Observa-se que os limitantes superiores encontrados pelo caso dedicado $(F \max 1)$ foram fixados em $100 \% \mathrm{e}$ a partir desses valores, calculam-se os valores referente aos demais níveis de flexibilidade.

Observe que os resultados são apresentados em relação aos limitantes superiores, e o valor de flexibilidade é medido como um decréscimo percentual na função objetivo em comparação com o caso dedicado. Os resultados computacionais mostram que o valor da flexibilidade depende do nível de flexibilidade (número de ligações entre itens e plantas) e do nível de capacidade. Em geral, para a maioria as configurações de flexibilidade, o valor da flexibilidade é maior para as instâncias com níveis de capacidade média (iguais a 90 e 100) e chegam a aproximadamente 14,5\% para as instâncias com 4 plantas e 4 produtos e 11, $5 \%$ para as instâncias com 6 plantas e 6 produtos. Isto indica que investir em flexibilidade é especialmente importante para estes casos. Para níveis de capacidade apertados e altos, o valos da flexibilidade é sempre menor do que $1 \%$. Em relação as configurações de flexibilidade, nota-se que os limitantes superiores encontrados com $20 \%$ da quantidade total de ligações ( Fmax 2 ) são substancialmente maiores do que os encontrados com as configurações que consideram $50 \%$ e $80 \%$ da quantidade total de ligações ( $F \max 3$ e $F \max 4$ ), especialmente para níveis de capacidade apertados e médios. No entanto, os resultados de $F \max 3$ e $F \max 4$ são similares para praticamente todos os níveis de capacidades.

A Tabela 2 mostra ainda que para as instâncias com 4 produtos, $50 \%$ da quantidade total de ligações $(F \max 3)$ é suficiente para encontrar praticamente todos os benefícios da flexibilidade total. Note que para este nível de flexibilidade, a diferença média em relação a flexibilitade total (Fmax 5$)$ é de apenas $0,14 \%$, o que evidencia que não é necessário que as plantas sejam muito flexíveis, ou seja, precisem produzir muitos tipos diferentes de produtos. Para as instâncias com 6 produtos, os gaps apresentados pelas configurações de flexibilidade (Fmax 2, Fmax 3, Fmax 4 e Fmax 5) para níveis de capacidade muito apertados (80 e 90) são grandes (Tabela 3), o que faz com que essas configurações apresentem limitantes superiores maiores do que $100 \%$. Para os demais níveis de capacidade observa-se novamente que com $50 \%$ da quantidade total de ligações encontra-se benefícios similares comparados com a flexibilidade total. Observa-se ainda que para 
essas instâncias, a média dos limitantes superiores encontrados com a configurção $F \max 4$ é um pouco menor do que o encontrado pela flexibilidade total ( $F \max 5)$. Isso ocorre porque os gaps encontrados pela configuração $F \max 5$ são maiores do que os encontrados pela $F \max 4$, principalmente para o nível de capacidade igual a 90.

Estes resultados estão alinhados e estendem as conclusões apresentadas em Fiorotto et al. (2018). Em Fiorotto et al. (2018) os autores concluem que, de maneira geral, uma quantidade de flexibilidade menor do que a quantidade necessária para construir o princípio da cadeia é suficiente para encontrar praticamente todos os benefícios da flexibilidade total para o problema de dimensionamento de lotes com máquinas paralelas. Neste estudo os resultados mostram que para o problema abordado, com $50 \%$ da quantidade total de ligações encontra-se benefícios similares comparados com a flexibilidade total.

Em relação a utilização de capacidade, a Tabela 2 mostra que os valores são similares para todas configurações analisadas. Além disso, os valores acima de $100 \%$ estão relacionados à utilização de hora extra. Note que para os casos com capacidade reduzida, a capacidade utilizada é superior à disponível no período em questão.

Os resultados evidenciam que do ponto de vista da prática industrial, para o objetivo de encontrar um planejamento que minimize os custos operacionais, as plantas não precisam produzir todos os produtos que compõem suas linhas de produção. Apenas uma pequena quantidade de produtos produzidos em cada uma das plantas, se distribuídos de forma eficiente, é suficiente para encontrar planos de produção que minimizem esses custos. Portanto, como investir em flexibilidade (adicionar novos produtos à linha de produção) pode ser, na prática, muito caro, esse estudo pode servir como direcionamento para que as indútrias realizem um planejamento adequado de investimento em flexibilidade para diminuir seus custos operacionais. Observa-se que essa economia de investimento pode fazer com que essas indústrias possam, uma vez que com menos gastos operacionais, investir em melhores preços de seus produtos, mantendo sua competitividade diante de um mercado cada vez mais competitivo e globalizado.

Tabela 2: Comparação dos limitantes superiores (em \%) e utilização de capacidade (em $\%)$ para diferentes níveis de flexibilidade.

\begin{tabular}{|c|c|c|c|c|c|c|c|c|c|c|c|}
\hline & & \multicolumn{2}{|c|}{ Fmax 1} & \multicolumn{2}{|c|}{ Fmax 2} & \multicolumn{2}{|c|}{ Fmax 3} & \multicolumn{2}{|c|}{ Fmax 4} & \multicolumn{2}{|c|}{ Fmax 5} \\
\hline produtos & Cap & $\mathrm{LS}(\%)$ & $\mathrm{UC}(\%)$ & LS(\%) & $\mathrm{UC}(\%)$ & $\mathrm{LS}(\%)$ & $\mathrm{UC}(\%)$ & $\operatorname{LS}(\%)$ & $\mathrm{UC}(\%)$ & $\mathrm{LS}(\%)$ & $\mathrm{UC}(\%)$ \\
\hline \multirow{5}{*}{4} & 80 & 100.00 & 114.60 & 100.00 & 114.60 & 99.96 & 114.58 & 100.04 & 114.57 & 99.99 & 114.58 \\
\hline & 90 & 100.00 & 101.86 & 99.98 & 101.86 & 89.20 & 101.99 & 88.94 & 101.99 & 89.15 & 102.00 \\
\hline & 100 & 100.00 & 91.68 & 99.78 & 91.68 & 85.76 & 91.73 & 85.56 & 91.73 & 85.53 & 91.74 \\
\hline & 110 & 100.00 & 83.34 & 99.74 & 83.34 & 99.34 & 83.34 & 99.15 & 83.34 & 99.12 & 83.34 \\
\hline & 120 & 100.00 & 76.40 & 99.74 & 76.40 & 99.35 & 76.40 & 99.15 & 76.40 & 99.12 & 76.40 \\
\hline Média & & 100.00 & 93.57 & 99.85 & 93.57 & 94.72 & 93.61 & 94.57 & 93.61 & 94.58 & 93.61 \\
\hline \multirow{5}{*}{6} & 80 & 100.00 & 114.30 & 101.74 & 114.31 & 104.48 & 114.63 & 101.14 & 114.33 & 101.05 & 114.32 \\
\hline & 90 & 100.00 & 101.60 & 105.45 & 101.66 & 93.18 & 101.90 & 89.35 & 101.83 & 90.77 & 101.84 \\
\hline & 100 & 100.00 & 91.44 & 89.17 & 91.48 & 88.75 & 91.48 & 88.59 & 91.49 & 88.58 & 91.49 \\
\hline & 110 & 100.00 & 83.13 & 99.63 & 83.13 & 99.26 & 83.13 & 99.17 & 83.13 & 99.16 & 83.13 \\
\hline & 120 & 100.00 & 76.20 & 99.62 & 76.20 & 99.27 & 76.20 & 99.17 & 76.20 & 99.18 & 76.20 \\
\hline Média & & 100.00 & 93.33 & 99.12 & 93.35 & 96.99 & 93.47 & 95.48 & 93.39 & 95.75 & 93.39 \\
\hline
\end{tabular}

Fonte: Elaborado pelos autores.

A Tabela 3 apresenta as médias dos gaps (Gap) e os tempos computacionais obtidos para cada conjunto de dados por nível de capacidade. A tabela mostra que o modelo é de difícil solução quando se considera níveis de flexibilidade diferentes da dedicada principalmente para o nível de capacidade 90, que é exatamente o nível em que a utilização de capacidade (UC\%) é próxima de $100 \%$, mostrando que este fato dificulta a resolução do problema.

Para as instâncias com 4 plantas e 4 produtos os gaps para as configurações Fmax 3 , $F \max 4$ e $F \max 5$ superam 10\% para nível de capacidade igual 90. Por outro lado, quando os níveis de capacidade são folgados, os gaps são praticamente zero. Para as instâncias com 6 plantas e 6 produtos os gaps são significativamente maiores e chegam a $29,52 \%$, de forma que o solver provou a otimalidade apenas para poucas instâncias, especificamente para níveis de capaci- 
dade iguais a 90 e 100. Observa-se que os esses resultados estão de acordo com o que é conhecido na literatura. Para capacidades apertadas e folgadas os benefícios da flexibilidade são próximos a zero, de forma que os problemas ficam mais fáceis de serem resolvidos, uma vez que as decisões de flexibilidade são menos importantes. Por outro lado, para capacidades médias, os benefícios da flexibilidade aumentam de forma significativa, o que torna os problemas mais difíceis de serem resolvidos, haja vista que as decisões de flexibilidade se tornam importantes. Isso explica a grande variação dos valores dos gaps para os níveis de capacidade iguais a 80, 90 e 100 (capacidades apertadas, médias e folgadas, respectivamente). Em relação aos tempos computacionais, observase que estes são muito baixos para todos níveis de capacidade para a configuração dedicada. No entanto, para as demais configurações os tempos computacionais apresentados são altos e para várias instâncias utiliza-se o tempo limite fixado (3600 segundos).

Tabela 3: Médias dos gaps (em \%) e tempos computacionais (em segundos) para diferentes níveis de flexibilidade.

\begin{tabular}{|c|c|c|c|c|c|c|c|c|c|c|c|}
\hline & & \multicolumn{2}{|c|}{ Fmax 1} & \multicolumn{2}{|c|}{ Fmax 2} & \multicolumn{2}{|c|}{ Fmax 3} & \multicolumn{2}{|c|}{ Fmax 4} & \multicolumn{2}{|c|}{ Fmax 5} \\
\hline produtos & Cap & $\operatorname{Gap}(\%)$ & $\mathrm{T}(\mathrm{s})$ & $\operatorname{Gap}(\%)$ & $\mathrm{T}(\mathrm{s})$ & $\operatorname{Gap}(\%)$ & $\mathrm{T}(\mathrm{s})$ & Gap (\%) & $\mathrm{T}(\mathrm{s})$ & Gap (\%) & $\mathrm{T}(\mathrm{s})$ \\
\hline \multirow{5}{*}{4} & 80 & 0.00 & 0.11 & 0.05 & 190.00 & 1.07 & 2872.35 & 1.09 & 2798.71 & 1.03 & 2786.15 \\
\hline & 90 & 0.00 & 0.12 & 0.01 & 194.69 & 12.67 & 3600.00 & 11.00 & 3600.00 & 11.11 & 3600.00 \\
\hline & 100 & 0.00 & 0.11 & 0.05 & 15.17 & 0.87 & 2832.32 & 1.06 & 2883.29 & 0.67 & 2493.99 \\
\hline & 110 & 0.00 & 0.12 & 0.01 & 4.27 & 0.05 & 57.65 & 0.03 & 26.03 & 0.05 & 10.67 \\
\hline & 120 & 0.01 & 0.11 & 0.01 & 4.27 & 0.05 & 93.33 & 0.03 & 79.74 & 0.06 & 13.73 \\
\hline Média & & 0.00 & 0.11 & 0.03 & 81.68 & 2.94 & 1891.13 & 2.64 & 1877.55 & 2.58 & 1780.91 \\
\hline \multirow{5}{*}{6} & 80 & 0.00 & 0.21 & 3.39 & 3600.00 & 5.75 & 3558.03 & 3.08 & 3479.36 & 2.94 & 3600.00 \\
\hline & 90 & 0.00 & 0.21 & 29.52 & 3600.00 & 26.61 & 3600.00 & 21.66 & 3600.00 & 23.31 & 3600.00 \\
\hline & 100 & 0.01 & 0.22 & 2.16 & 3600.00 & 2.21 & 3600.00 & 2.08 & 3600.00 & 1.91 & 3600.00 \\
\hline & 110 & 0.01 & 0.22 & 0.08 & 674.59 & 0.11 & 1008.97 & 0.10 & 608.96 & 0.09 & 347.55 \\
\hline & 120 & 0.01 & 0.22 & 0.09 & 879.47 & 0.14 & 1106.93 & 0.10 & 917.87 & 0.10 & 719.55 \\
\hline Média & & 0.01 & 0.21 & 7.05 & 2470.81 & 6.96 & 2574.79 & 5.40 & 2441.24 & 5.67 & 2373.42 \\
\hline
\end{tabular}

Fonte: Elaborado pelos autores.

Com o intuito de analisar melhor o conceito de flexibilidade para o problema de dimensionamento de lotes com custo de transporte e plantas flexíveis, as Tabelas 4 e 5 mostram informações a respeito da estrutura das soluções encontradas. Desta forma, apresenta-se as porcentagens que os custos de preparo (Prep.), hora extra (H.E.), transporte (Tran.) e estoque (Est.) assumem nas soluções obtidas em cada conjunto de dados, para todos os níveis de flexibilidade ( $F \max 1$, $F \max 2$ e $F \max 3$ na Tabela 4 e $F \max 4$ e $F \max 5$ na Tabela 5). Em geral, para todos os níveis de flexibilidade, com níveis de capacidade apertados, a porcentagem de custo de hora extra nas soluções é muito alta (acima de $80 \%$ ). Por outro lado, com o aumento do nível de capacidade, a porcentagem do custo de preparação aumenta e atinge valores acima de $74 \%$ quando a capacidade é igual a 120. Por fim, observa-se ainda que o aumento do nível de capacidade faz com que a porcentagem do custo de transporte aumente de forma significativa. Embora os valores brutos dos custos de transporte sejam reduzidos à medida que se aumenta o nível de flexibilidade, de acordo com a Tabela 4, observa-se que a porcentagem dos custos de transporte mantém sempre cerca de $1 / 3$ da porcentagem dos custos de preparação, independentemente do nível de capacidade e de flexibilidade. Isso indica que esse ponto de equilíbrio entre custos de preparação e custos de transporte se mantém estável independentemente do nível de capacidade e de flexibilidade.

Por fim, observa-se ainda que foram testadas instâncias maiores, com 12 produtos e 12 plantas. No entanto, o CPLEX não obteve boas soluções para estes casos para as configurações que consideram a possibilidade de flexibilidade, o que dificultou a análise do valor da flexibilidade. Dessa forma, conclui-se que para realizar a análise de flexibilidade em instâncias maiores faz-se necessário o desenvolvimento/aplicação de outros métodos de solução. Alguns exemplos de métodos que foram aplicados recentemente em problemas similares são: heurísticas de decomposição (Fiorotto et al., 2015; Wu et al., 2018) e a metaheurística kernel search (Carvalho e Nascimento, 2018). 
Tabela 4: Estrutura das soluções obtidas (porcentagem de cada custo no valor total da função objetivo).

\begin{tabular}{|c|c|c|c|c|c|c|c|c|c|c|c|c|c|}
\hline & & \multicolumn{4}{|c|}{ Fmax 1} & \multicolumn{4}{|c|}{ Fmax 2} & \multicolumn{4}{|c|}{ Fmax 3} \\
\hline produtos & Cap & Prep. & H.E. & Tran. & Est. & Prep. & H.E. & Tran. & Est. & Prep. & H.E. & Tran. & Est. \\
\hline \multirow{5}{*}{4} & 80 & 1.33 & 98.18 & 0.46 & 0.03 & 1.33 & 98.18 & 0.46 & 0.03 & 1.34 & 98.06 & 0.45 & 0.15 \\
\hline & 90 & 6.67 & 88.83 & 2.31 & 2.19 & 6.67 & 88.85 & 2.29 & 2.19 & 7.90 & 87.07 & 2.49 & 2.54 \\
\hline & 100 & 62.47 & 13.75 & 21.67 & 2.11 & 62.60 & 13.78 & 21.50 & 2.11 & 73.80 & 0.00 & 24.44 & 1.76 \\
\hline & 110 & 74.24 & 0.00 & 25.76 & 0.00 & 74.44 & 0.00 & 25.56 & 0.00 & 74.74 & 0.00 & 25.26 & 0.00 \\
\hline & 120 & 74.24 & 0.00 & 25.76 & 0.00 & 74.44 & 0.00 & 25.56 & 0.00 & 74.73 & 0.00 & 25.27 & 0.00 \\
\hline Média & & 43.79 & 40.15 & 15.19 & 0.87 & 43.89 & 40.16 & 15.08 & 0.87 & 46.50 & 37.03 & 15.58 & 0.89 \\
\hline \multirow{5}{*}{6} & 80 & 1.39 & 98.11 & 0.47 & 0.03 & 1.38 & 98.03 & 0.45 & 0.14 & 1.55 & 97.07 & 0.41 & 0.96 \\
\hline & 90 & 7.33 & 87.82 & 2.45 & 2.40 & 7.13 & 88.23 & 2.30 & 2.34 & 8.73 & 84.58 & 2.45 & 4.24 \\
\hline & 100 & 65.39 & 10.82 & 21.89 & 1.89 & 74.10 & 0.00 & 24.19 & 1.71 & 74.50 & 0.00 & 23.86 & 1.65 \\
\hline & 110 & 74.92 & 0.00 & 25.08 & 0.00 & 75.20 & 0.00 & 24.80 & 0.00 & 75.48 & 0.00 & 24.52 & 0.00 \\
\hline & 120 & 74.92 & 0.00 & 25.08 & 0.00 & 75.21 & 0.00 & 24.79 & 0.00 & 75.47 & 0.00 & 24.53 & 0.00 \\
\hline Média & & 44.79 & 39.35 & 14.99 & 0.87 & 46.60 & 37.25 & 15.31 & 0.84 & 47.14 & 36.33 & 15.15 & 1.37 \\
\hline
\end{tabular}

Fonte: Elaborado pelos autores.

Tabela 5: Estrutura das soluções obtidas (porcentagem de cada custo no valor total da função objetivo).

\begin{tabular}{|c|c|c|c|c|c|c|c|c|c|}
\hline & & \multicolumn{9}{|c|}{ Fmax 4 } & \multicolumn{4}{c|}{ Fmax 5 } \\
\hline produtos & Cap & Prep. & H.E. & Tran. & Est. & Prep. & H.E. & Tran. & Est. \\
\hline \multirow{4}{*}{4} & 80 & 1.35 & 97.92 & 0.44 & 0.29 & 1.34 & 97.97 & 0.44 & 0.25 \\
& 90 & 7.92 & 86.97 & 2.44 & 2.67 & 7.92 & 87.13 & 2.43 & 2.52 \\
& 100 & 74.08 & 0.00 & 24.28 & 1.65 & 74.15 & 0.00 & 24.24 & 1.61 \\
& 110 & 74.88 & 0.00 & 25.12 & 0.00 & 74.90 & 0.00 & 25.10 & 0.00 \\
& 120 & 74.88 & 0.00 & 25.12 & 0.00 & 74.91 & 0.00 & 25.09 & 0.00 \\
\hline Média & & 46.62 & 36.98 & 15.48 & 0.92 & 46.64 & 37.02 & 15.46 & 0.88 \\
\hline \multirow{4}{*}{6} & 80 & 1.43 & 97.15 & 0.43 & 1.00 & 1.43 & 97.15 & 0.42 & 1.00 \\
& 90 & 8.94 & 84.87 & 2.56 & 3.63 & 8.89 & 84.95 & 2.52 & 3.65 \\
& 100 & 74.74 & 0.00 & 23.72 & 1.54 & 74.72 & 0.00 & 23.71 & 1.57 \\
& 110 & 75.55 & 0.00 & 24.45 & 0.00 & 75.55 & 0.00 & 24.45 & 0.00 \\
& 120 & 75.55 & 0.00 & 24.45 & 0.00 & 75.54 & 0.00 & 24.46 & 0.00 \\
\hline Média & & 47.24 & 36.40 & 15.12 & 1.23 & 47.23 & 36.42 & 15.11 & 1.24 \\
\hline
\end{tabular}

Fonte: Elaborado pelos autores.

\section{Conclusões e Propostas Futuras}

Neste artigo um modelo matemático para o problema de dimensionamento de lotes com plantas flexíveis e custo de transporte é proposto e uma análise computacional com relação ao valor da flexibilidade das plantas é realizada, em que o objetivo consiste em verificar se com uma quantidade limitada de flexibilidade pode-se encontrar todos os benefícios da flexibilidade total. Foram analisados um total de 1000 instâncias adaptadas da literatura, onde os testes computacionais foram obtidos a partir da utilização do pacote CPLEX 12.9 para todos os níveis de flexibilidade envolvidos com critérios de parada de gap igual ou inferior a $0,1 \%$ ou tempo limite de 3600 segundos. Os resultados computacionais mostraram que adicionando flexibilidade, houve uma nítida melhoria dos valores das soluções obtidas a partir dos níveis de capacidade superiores a 100 para instâncias com 6 plantas 6 produtos e uma melhor performance em todos os níveis de capacidade para instâncias com 4 plantas e 4 produtos, comparado ao caso de plantas dedicadas, o que evidencia a importância de se montar sistemas flexíveis. Além disso, observou-se que é necessário apenas $50 \%$ da quantidade total de ligações para se obter praticamente os mesmos benefícios da flexibilidade total.

Do ponto de vista da prática industrial, esses resultados mostran que, as plantas não precisam produzir todos os produtos que compõem suas linhas de produção. Apenas uma pequena quantidade de produtos produzidos em cada uma das plantas, se distribuídos de forma eficiente, é suficiente para encontrar planos de produção que minimizem os custos operacionais. Portanto, como investir em flexibilidade (adicionar novos produtos à linha de produção) pode ser, na prática, muito caro, esse estudo pode servir como direcionamento para que as indútrias realizem um pla- 
nejamento adequado de investimento de flexibilidade para diminuir seus custos operacionais. Por fim, observa-se ainda, que a dificuldade de resolução do modelo prejudicou uma análise precisa sobre flexibilidade, para instâncias com 6 plantas e 6 produtos e capacidade apertada, pois o valor dos gaps apresentados pelas configurações de flexibilidade foram altos.

Como propostas futuras, observa-se que a ideia de analisar a flexibilidade de um modelo matemático vem acoplado à diversas outras análises de aspectos relacionados ao modelo que podem ser realizadas, como por exemplo, a que nível o custo de hora extra pode interferir no limitante superior nos diferentes níveis de flexibilidade, bem como os custos de estoque e a homogeneidade da demanda. Inclusive, é interessante levar em consideração a utilização de atraso no modelo, sendo assim dispensável o uso de hora extra, neste caso a definição da variável de produção deve ser modificada possibilitando o atraso e o estoque no último período do horizonte de planejamento, o que pode ser interessante para a análise de custos variáveis para atraso. Os altos valores de gap, bem como os altos tempos computacionais sugerem ainda a necessidade de desenvolver métodos de solução para resolver formulação e realizar uma análise de flexibilidade para sistemas maiores.

Agradecimentos. Este trabalho teve o apoio financeiro da CAPES, CNPq (processos números 405048/2018-1 e 305261/2018-5), FAPESP (processos números 2013/07375-0, 2018/18754-5 e 2016/01860-1) e FAEPEX (processo número 2030/19).

\section{Referências}

Belo-Filho, M. A. F., Toledo, F. M. B. e Almada-Lobo, B. Models for capacitated lot-sizing problem with backlogging, setup carryover and crossover. Journal of the Operational Research Society, v. 65, n. 11, p. 1735-1747, 2014.

Bertrand, J. W. M. Supply chain design: flexibility considerations. In: De Kok, A., Graves, S. (Eds.), Supply Chain Management: Design, Coordination and Operation, Hand-books in Operations Research and Management Science. Elsevier, North-Holland, v. 11, p. 133-198, 2003.

Bhatnagar, R., Chandra, P. e Goyal, S. K. Models for multi-plant coordination. European Journal of Operational Research, v. 67, n. 2, p. 141-160, 1993.

Carreno, J. J. Economic lot scheduling for multiple products on parallel identical processors. Management Science, v. 36, n. 3, p. 348-358, 1990.

Carvalho, D. M. e Nascimento, M. C. V. Lagrangian heuristics for the capacitated multi-plant lot sizing problem with multiple periods and items. Computers \& Operations Research, v. 71, p. 137-148, 2016.

Carvalho, D. M. e Nascimento, M. C. V. A kernel search to the multi-plant capacitated lot sizing problem with setup carry-over. Computers \& Operations Research, v. 100, p. 43-53, 2018.

de Matta, R. e Miller, T. Production and inter-facility transportation scheduling for a process industry. European Journal of Operational Research, v. 158, n. 1, p. 72-88, 2004.

Denizel, M. e Süral, H. On alternative mixed integer programming formulations and lp-based heuristics for lot-sizing with setup times. Journal of the Operational Research Society, v. 57, p. 389-399, 2006.

Fine, C. H. e Freund, R. M. Optimal investment in product-flexible manufacturing capacity. $M a-$ nagement Science, v. 36, n. 4, p. 449-466, 1990.

Fiorotto, D. J. e de Araujo, S. A. Reformulation and a lagrangian heuristic for lot sizing problem on parallel machines. Annals of Operations Research, v. 217, n. 1, p. 213-231, 2014. 
Fiorotto, D. J., de Araujo, S. A. e Jans, R. Hybrid methods for lot sizing on parallel machines. Computers \& Operations Research, v. 63, p. 136-148, 2015.

Fiorotto, D. J., del Huaccha Neyra, J. e de Araujo, S. A. Impact analysis of setup carryover and crossover on lot sizing problems. International Journal of Production Research, v. , 2019. doi:10.1080/00207543.2019.1680892.

Fiorotto, D. J., Jans, R. e de Araujo, S. A. An analysis of formulations for the capacitated lot sizing problem with setup crossover. Computers \& Industrial Engineering, v. 106, p. 338-350, 2017.

Fiorotto, D. J., Jans, R. e de Araujo, S. A. Process flexibility and the chaining principle in lot sizing problems. International Journal of Production Economics, v. 204, p. 244-263, 2018.

Gerwin, D. Manufacturing flexibility: a strategic perspective. Management Science, v. 39, n. 4, p. 395-410, 1993.

Graves, S. C. e Tomlin, B. T. Process flexibility in supply chains. Management Science, v. 49, n. 7, p. 907-919, 2003.

Guimaraes, L., Klabjan, D. e Almada-Lobor, B. Annual production budget in the beverage industry. Engineering Applications of Artificial Intelligence, v. 25, n. 2, p. 229-241, 2012.

Gurumurthi, S. e Benjaafar, S. Modeling and analysis of flexible queueing systems. Naval Research Logistics, v. 51, n. 5, p. 755-782, 2004.

Jans, R. Solving lot-sizing problems on parallel identical machines using symmetry-breaking constraints. INFORMS Journal on Computing, v. 21, n. 1, p. 123-136, 2009.

Jans, R. e Degraeve, Z. An industrial extension of the discrete lot sizing and scheduling problem. IEE Transactions, v. 36, n. 1, p. 47-58, 2004.

Johnzén, C., Dauzère-Pérès, S. e Vialletelle, P. Flexibility measures for qualification management in wafer fabs. Production Planning \& Control, v. 22, n. 1, p. 81-90, 2011.

Jordan, W. C. e Graves, S. C. Principles on the benefits of manufacturing process flexibility. Management Science, v. 41, n. 4, p. 577-594, 1995.

Koste, L. L. e Malhotra, M. K. A theoretical framework for analyzing the dimensions of manufacturing flexibility. Journal of Operations Management, v. 18, n. 1, p. 75-93, 1999.

Mak, H.-Y. e Shen, Z.-J. M. Stochastic programming approach to process flexibility design. Flexible Services and Manufacturing Journal, v. 21, n. 3-4, p. 75-91, 2009.

Mateus, G. R., Ravetti, M. G., de Souza, M. C. e Valeriano, T. M. Capacitated lot sizing and sequence dependent setup scheduling: an iterative approach for integration. Journal of Scheduling, v. 13 , n. 3, p. 245-259, 2010.

Melega, G. M., Fiorotto, D. J. e de Araujoo, S. A. Formulações fortes para o problema de dimensionamento de lotes com várias plantas. TEMA - Tendências em Matemática Aplicada e Computacional, v. 14, n. 3, p. 305-318, 2013.

Melo, R. A. e Wolsey, L. A. Mip formulation and heuristics for two-level productiontransportation problems. Computers \& Operations Research, v. 39, n. 11, p. 2776-2786, 2012.

Muriel, A., Somasundaram, A. e Zhang, Y. Impact of partial manufacturing flexibility on production variability. Manufacturing \& Service Operations Management, v. 8, n. 2, p. 192-205, 2006. 
Pérez-Pérez, M., Bedia, A.-M. S., López-Fernández, M.-C. e García-Piqueres, G. Research opportunities on manufacturing flexibility domain: A review and theorybased research agenda. Journal of Manufacturing Systems, v. 48, Part A, p. 9-20, 2018.

Rowshannahad, M., Dauzère-Pérès, S. e Cassini, B. Capacitated qualification management in semiconductor manufacturing. Omega, v. 54, p. 50-59, 2015.

Sambasivan, M. e Yahya, S. A lagrangean-based heuristic for multi-plant, multi-item, multi-period capacitated lot-sizing problems with inter-plant transfers. Computers \& Operations Research, v. 32 , n. 3, p. 537-555, 2005.

Tang, C. S. e Denardo, E. V. Models arising from a flexible manufacturing machine, part i: Minimization of the number of tool switches. Operations Research, v. 36, n. 5, p. 767-777, 1988.

Teixeira, S. B. Análise de flexibilidade para o problema de dimensionamento de lotes com plantas flexíveis e custo de transporte. $69 \mathrm{f}$. Dissertação (Mestrado em Matemática) - Instituto de Biociências, Letras e Matemática, Universidade Estadual Paulista "Júlio de Mesquita Filho", São José do Rio Preto-SP, 2019.

Toledo, F. M. B. e Armentano, V. A. A lagrangian-based heuristic for the capacitated lot-sizing problem in parallel machines. European Journal of Operational Research, v. 175, n. 2, p. 10701083, 2006.

Trigeiro, W. W., Thomas, L. J. e McClain, J. O. Capacitated lot sizing with setup times. Management science, v. 35, n. 3, p. 353-366, 1989.

Vincent, B., Duhamel, C., Ren, L. e Tchernev, N. A population-based metaheuristic for the capacitated lot-sizing problem with unrelated parallel machines. International Journal of Production Research, 2020. doi: 10.1080/00207543.2019.1685699.

Wu, T., Liang, Z. e Zhang, C. Analytics branching and selection for the capacitated multi-item lot sizing problem with nonidentical machines. INFORMS Journal on Computing, v. 30, n. 2, p. 236-258, 2018.

Xiao, J., Yang, H., Zhang, C., Zheng, L. e Gupta, J. N. A hybrid lagrangian-simulated annealingbased heuristic for the parallel-machine capacitated lot-sizing and scheduling problem with sequence-dependent setup times. Computers \& Operations Research, v. 63, p. 72-82, 2015. 